\title{
Corticosteroid-induced osteoporosis prevention: longitudinal practice patterns in The Netherlands 2001-2005
}

\author{
M. Duyvendak • M. Naunton • J. Atthobari • \\ P. B. van den Berg • J. R. B. J. Brouwers
}

Received: 13 November 2006 / Accepted: 22 January 2007 / Published online: 24 February 2007

(C) International Osteoporosis Foundation and National Osteoporosis Foundation 2007

\begin{abstract}
Summary We investigated prevention trends and predictors for osteoporosis prevention in long term corticosteroid users. The use of bisphosphonates increased from 2001 to 2005. Longer duration of corticosteroid use and DMARD use were predictors for receiving prevention. Females appear reasonably well treated; however, men require more attention.

Introduction Previous studies have shown that long-term corticosteroid users are undertreated for osteoporosis prevention. Our aim was to identify prevention trends in long-term corticosteroid users from 2001-2005 in The Netherlands and to identify predictors for bisphosphonate prophylaxis.

Methods Pharmacy dispensing data were used from 9 community pharmacies. All oral corticosteroid doses were converted to "prednisolone equivalents". We then identified long-term ( $\geq 90$ days) corticosteroid episodes, which required bisphosphonate prophylaxis as per 2002 Dutch
\end{abstract}

M. Duyvendak · M. Naunton · J. R. B. J. Brouwers

Department of Clinical Pharmacy,

Tjongerschans Hospital,

Heerenveen, The Netherlands

M. Duyvendak $(\varangle) \cdot$ M. Naunton $\cdot$ J. Atthobari $\cdot$

P. B. van den Berg · J. R. B. J. Brouwers

Department of Pharmacotherapy and Pharmaceutical Care,

University of Groningen,

Antonius Deusinglaan 1,

9713 AV Groningen, The Netherlands

e-mail: mduyvendak@tjongerschans.nl

M. Naunton

School of Science, Charles Darwin University,

Northern Territory, Australia

J. Atthobari · P. B. van den Berg • J. R. B. J. Brouwers Groningen University Institute for Drug Exploration (GUIDE),

Groningen, The Netherlands guidelines; Multivariate logistic regression was used to identify predictors for receiving prevention.

Results We identified 615 different corticosteroid patients requiring prophylaxis. From 2001-2005 the use of bisphosphonates increased from $38 \%$ to $54 \%(\mathrm{p}=0.001)$. In 2005 females were prescribed more bisphosphonates than males $(61 \%$ vs. $39 \% ; p=0.002)$, or any treatment $(72 \%$ vs. $45 \% ; \mathrm{p}<0.001)$. Multivariate analysis showed that longer duration of corticosteroid use and disease-modifying antirheumatic drug (DMARD) use were independent predictors of bisphosphonate use. Use of respiratory medication was a negative predictor of bisphosphonate use.

Conclusion There has been a significant increase in osteoporosis prophylaxis in a population at high risk for osteoporosis/fractures. In particular, females appear reasonably well treated; however, men are still not receiving prevention to the same degree as women.

Keywords Corticosteroids · Osteoporosis .

Prescribing patterns $\cdot$ Prevention $\cdot$ The Netherlands

\section{Introduction}

Corticosteroid-induced osteoporosis (CIOP) is the most common type of secondary osteoporosis with up to $50 \%$ of chronic corticosteroid users sustaining fractures $[1,2]$. Numerous international studies have demonstrated that osteoporosis is underrecognised and undertreated in corticosteroid users [3-5]. In the Netherlands, national specialist guidelines (2002) recommend preventive therapy for those on $\geq 7.5 \mathrm{mg}$ for longer than 3 months [6]. Curtis et al. [3] recently showed that despite significant increases in osteoporosis prevention in long-term corticosteroid users, absolute rates remain low in the USA. From a European perspective, 
we are unaware of any published research investigating the longitudinal patterns in osteoporosis prescribing.

The aim of this study was to (a) identify prevention trends in long-term corticosteroid users who required osteoporosis/fracture prophylaxis as per Dutch 2002 guidelines from July 2001-December 2005 (b) identify predictors for receiving bisphosphonate prophylaxis.

\section{Methods}

Population

We used pharmacy dispensing data (deidentified) from nine community pharmacies spread over four cities/towns in the northern part of The Netherlands (catchment population approximately 125,000). To assess the prevalence of prophylaxis for CIOP between July 2001 and December 2005, we determined for each half year period whether a patient required prophylaxis with bisphosphonates during that period and whether they were prescribed a bisphosphonate or other type of prevention.

\section{Inclusion criteria}

We identified all patients ( $>18$ years) receiving oral corticosteroids for $\geq 90$ days requiring prophylaxis accord- ing to Dutch (2002) guidelines [6] during the period July 2001 to December 2005. Specifically, the guidelines recommend that men $\geq 70$ years old and postmenopausal women who receive $>7.5 \mathrm{mg}-15 \mathrm{mg}$ /day of prednisolone (or equivalent) or $>15 \mathrm{mg} /$ day prednisolone (or equivalent) independent of age and gender should receive bisphosphonate treatment independent of BMD. The guidelines state that calcium and/or vitamin D alone is insufficient prophylaxis. A postmenopausal status was assumed for women aged $\geq 55$ years.

Qualifying corticosteroid medications

Corticosteroid episodes were identified by selecting all prescriptions with a maximum of 90 days between the end and start of two consecutive prescriptions. We qualified all corticosteroids with the anatomical therapeutic chemical classification (ATC code) H02AB. Injectable, inhaled, and topical corticosteroids were excluded. All corticosteroid doses were converted to "prednisolone equivalents" using defined daily dose (DDD) [7]. The average observed daily dose for each subject was calculated by dividing the cumulative dose dispensed by the number of days of the total corticosteroid episode. We excluded those who appeared to use implausible daily doses of prednisolone ( $\geq 100 \mathrm{mg} /$ day prednisolone or equivalent [3]). The initial prescriber of the corticosteroid was determined from the
Fig. 1 Flowchart of patient inclusion

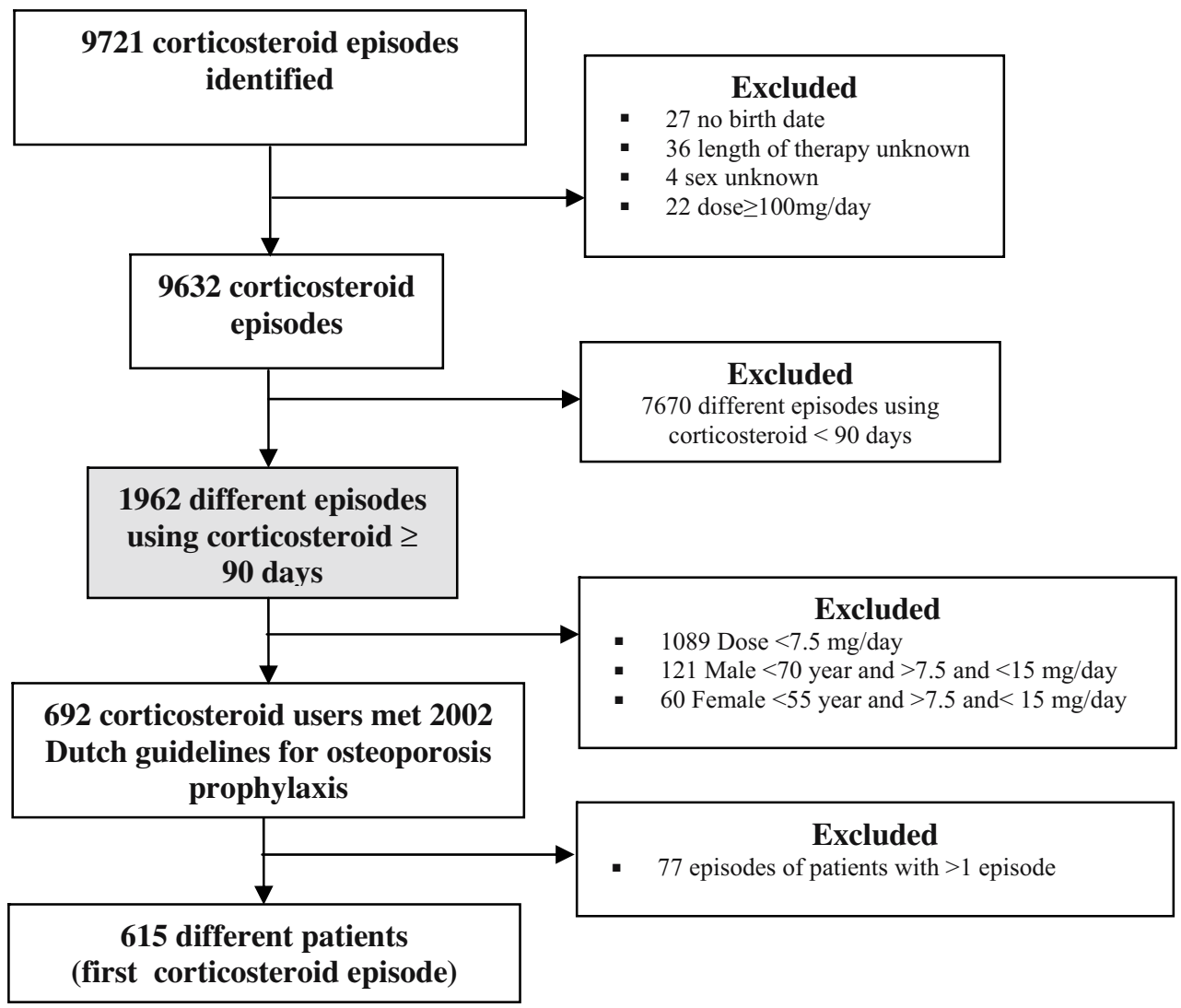


pharmacy data; in The Netherlands both general practitioners (GPs) and specialists prescribe directly.

\section{Co-medication}

All co-medications (excluding antibiotics) during the corticosteroid episode were calculated. Concurrent respiratory medications were: inhaled corticosteroids, short/long acting inhaled $\beta_{2}$ agonists, or leukotriene-receptor antagonists. Other concurrent medication included: disease-modifying anti-rheumatic drugs (DMARDs), proton pump inhibitors (PPI), non-steroidal anti-inflammatory drugs (NSAIDs), antiplatelets, and vitamin $\mathrm{K}$ antagonists (VKA) or low molecular weight heparins (LMWH).

\section{Statistics}

Standard descriptive methods and statistics were used to characterise the study population using SPSS ${ }^{\circledR}$ version 14 (SPSS, Chicago, IL United States). A logistic regression model was used to identify factors significantly associated with the prescribing of a bisphosphonate. Univariate and multivariate analyses were used to predict which variables (age; sex; number of co-medication; duration of corticosteroid use; prescriber of corticosteroid; daily corticosteroid dose; concomitant PPI, NSAID, DMARD, respiratory medication, antiplatelets, VKA, and LMWH use) influenced the prescribing of a bisphosphonate. For the multivariate analysis each individual variable was adjusted for the remaining variables in the model as aforementioned. A two-tailed $p$ value $\leq 0.05$ was considered statistically significant.

\section{Results}

We identified 692 different corticosteroid episodes which met the inclusion criteria for requiring prophylaxis. This group consisted of 615 different patients (Fig. 1). Of these 615 patients requiring prophylaxis, 267 (43\%) received a bisphosphonate (51\% received any prevention). The characteristics of the bisphosphonate and non-bisphosphonate users are shown in Table 1.

Trends in osteoporosis prevention

Figure 2a shows the use of CIOP-prophylaxis in patients who require prophylaxis with bisphosphonates according to Dutch guidelines. From 2001-2005 bisphosphonate use increased from $38 \%$ to $54 \%$ (trend $\mathrm{p}=0.001$ ) and any treatment increased from $48 \%$ to $62 \%$ (trend $p=0.001$ ).

Figure $2 \mathrm{~b}$ shows the sex differences in osteoporosis prevention (2001-2005) in those requiring prophylaxis. Females received more osteoporosis prevention than males
Table 1 Characteristics of 615 corticosteroid users (July 2001-Dec 2005) requiring bisphosphonate prophylaxis according to 2002 Dutch guidelines

\begin{tabular}{|c|c|c|c|}
\hline Characteristic & $\begin{array}{l}\text { Bisphosphonate } \\
\text { prescribed } \\
(\mathrm{n}=267)\end{array}$ & $\begin{array}{l}\text { Bisphosphonate } \\
\text { not prescribed } \\
(\mathrm{n}=348)\end{array}$ & Statistics \\
\hline Female (\%) & $187(70)$ & $188(54)$ & $\mathrm{p}<0.001$ \\
\hline Mean age (SD) & $66.5(14.2)$ & $69.7(16.1)$ & $\mathrm{p}=0.01$ \\
\hline Mean dose (SD) & $16.3(9.6)$ & $15.5(9.3)$ & $\mathrm{p}=0.31$ \\
\hline 7.5-15 (\%) & $158(59)$ & $218(63)$ & \\
\hline$>15$ & $109(41)$ & $130(37)$ & \\
\hline $\begin{array}{l}\text { Mean length of } \\
\text { therapy (days; SD) }\end{array}$ & $631(517)$ & $341(348)$ & $\mathrm{p}<0.001$ \\
\hline$>180$ days $(\%)$ & $222(83)$ & $192(55)$ & $\mathrm{p}<0.001$ \\
\hline $\begin{array}{l}\text { Prescriber of initial } \\
\text { corticosteroid* }(\%)\end{array}$ & & & $\mathrm{p}=0.07$ \\
\hline GP & $90(47)$ & $157(55)$ & \\
\hline Specialist & $103(53)$ & $128(45)$ & \\
\hline $\begin{array}{l}\text { Mean number of } \\
\text { co-medications** }(\mathrm{SD})\end{array}$ & $5.6(3.9)$ & $5.3(3.6)$ & $\mathrm{p}=0.28$ \\
\hline PPI (\%) & $164(61)$ & $177(51)$ & $\mathrm{p}=0.009$ \\
\hline NSAID & $133(50)$ & $122(35)$ & $\mathrm{p}<0.001$ \\
\hline Antiplatelets & $53(20)$ & $69(20)$ & $\mathrm{p}=0.99$ \\
\hline VKA/LMWH & $53(20)$ & $83(24)$ & $\mathrm{p}=0.24$ \\
\hline Resp & $83(31)$ & $156(45)$ & $\mathrm{p}=0.001$ \\
\hline DMARDs & 77 (29) & $33(9)$ & $\mathrm{p}<0.001$ \\
\hline \multicolumn{4}{|l|}{$\begin{array}{l}\text { Osteoporosis } \\
\text { prevention (\%) }\end{array}$} \\
\hline Calcium & $105(39)$ & $24(7)$ & $\mathrm{p}<0.001$ \\
\hline Vitamin D & $14(5)$ & $5(1)$ & $\mathrm{p}=0.01$ \\
\hline $\begin{array}{l}\text { Calcium and } \\
\text { vitamin } \mathrm{D}^{\mathrm{b}}\end{array}$ & $67(25)$ & $12(3)$ & $\mathrm{p}<0.001$ \\
\hline HRT & $4(1)$ & $7(2)$ & $\mathrm{p}=0.76$ \\
\hline SERMs & 0 & 0 & NA \\
\hline
\end{tabular}

${ }^{\mathrm{a}}$ bisphosphonate vs. non bisphosphonate user; *three prescribers unknown +134 prevalent corticosteroid users (initial prescriber not retraceable) $* *$ excluding the corticosteroid; $\mathrm{GP}=$ general practitioner; PPI $=$ proton pump inhibitors; NSAIDs $=$ non-steroidal antiinflammatory drugs; VKA = vitamin $\mathrm{K}$ antagonists; LMWH = low molecular weight heparins; Resp = inhaled medications for asthma/ COPD; DMARDs = disease-modifying anti-rheumatic drugs; $H R T=$ oral estrogen \pm progesterone; $\mathrm{SERMs}=$ selective estrogen receptor modulators

${ }^{\mathrm{b}}$ includes activated and non-activated vitamin D

( $72 \%$ vs. $45 \% ; \mathrm{p}<0.001)$ in 2005 ; specifically, females used more bisphosphonates in 2005 than males (61\% vs. 39\%; $\mathrm{p}=0.002)$. Bisphosphonate use in females significantly increased from $40 \%$ in 2001 to $61 \%$ in 2005 ( $p=0.001$ ). For men there was a non-significant increase in any osteoporosis prevention $(\mathrm{p}=0.09)$ or bisphosphonate use $(\mathrm{p}=0.20)$.

Variables influencing the use of bisphosphonates

Multivariate analysis showed that duration of corticosteroid use $>360$ days (OR 6.50; 95\%CI:3.64-11.60) and DMARD 
Fig. 2 Trends in prescribing of osteoporosis prophylaxis in corticosteroid episodes requiring bisphosphonates as per Dutch guidelines. a) All preventive agents b) Influence of gender a

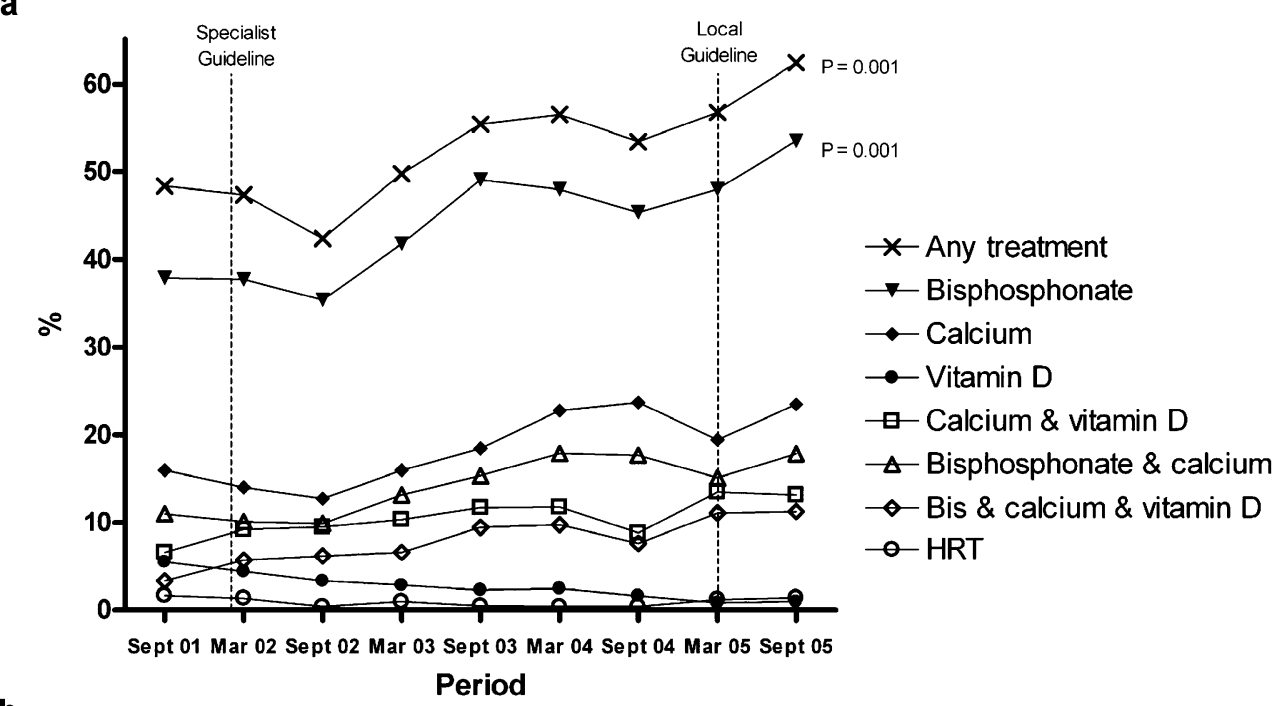

b

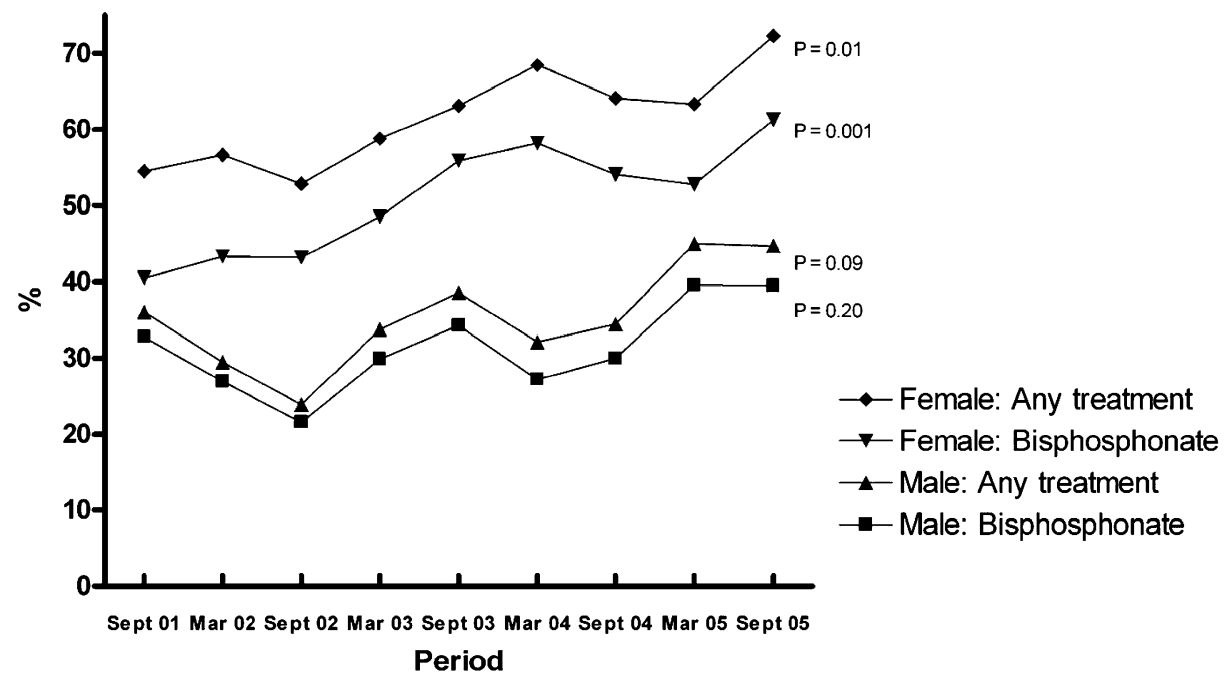

use (OR 2.55; 95\%CI:1.38-4.69) were positive predictors to the prescribing of bisphosphonates. Use of respiratory medication (OR 0.48; 95\%CI:0.29-0.78) was a negative predictor for the prescribing of bisphosphonates. Male sex was a negative predictor in the univariate analysis (OR 0.51 ; 95\% CI:0.37-0.72), but in multivariate analysis trended towards significance (OR 0.69; 95\%CI:0.44-1.09).

\section{Discussion}

Our study has described the trends in osteoporosis prevention in a group of patients in which Dutch guidelines recommend bisphosphonates be used independent of BMD. We have observed improvements in preventive therapy use in those who fulfil Dutch guidelines. These improvements are likely to have coincided with the introduction of specialist Dutch guidelines for osteoporosis and CIOP in 2002. In addition, in March 2005, we distributed locally produced CIOP prevention guidelines to family physicians, pharmacists, and specialists. Curtis et al. [3] recently showed that in the USA there has been a gradual increase in BMD testing and osteoporosis prophylaxis prescribing in corticosteroid users, although absolute rates remain low.

Disappointingly we have shown that males are still significantly less likely to receive osteoporosis prophylaxis than females. Furthermore, in contrast to females, males showed no significant improvement from 2001-2005. Other recent studies have also found that males appear undertreated more than females [3, 8]. It is possible that females are given more attention than males because of their postmenopausal bone loss, which men do not experience. However, the reasons why men are less treated remain enigmatic and further study is required to investigate these sex differences.

DMARD users were 2.5 times more likely to receive a bisphosphonate and users of respiratory medication were 
less than half as likely to receive bisphosphonate prophylaxis. We speculate that this may be related to the particular doctor specialty. If this is true, our data is in agreement with the recent paper published by Saag et al. [8] who found rheumatologists were 3.5 times more likely to prescribe non-estrogen osteoporosis therapy and respiratory physicians the least likely out of all specialists to prescribe non-estrogen osteoporosis prevention in long-term corticosteroid users.

There are some limitations to our study that require comment. Firstly, we did not have clinical information on our patient group such as BMD testing or fracture history. However, Dutch guidelines recommended all patients commence biphosphonates if they are on $>15 \mathrm{mg}$ or are receiving $>7.5 \mathrm{mg} /$ day and are postmenopausal or $>70$ years (male) independent of BMD. We were also unable to exclude those patients in whom osteoporosis prevention may be inappropriate, for example, patients suffering from alcoholism, dementia, and serious renal impairment. However, given the mean age of our patients we believe this is also unlikely to affect our overall results. Lastly, we have obtained data from a specific region (and do not have a control group) in The Netherlands and therefore our results may not be generalisable to the entire Dutch population. However, corticosteroid use in our catchment population $(0.5 \%)$ was comparable to other population-based studies $(0.5-0.9 \%)[9,10]$ and our exclusion criteria were negligible.

Despite our study's limitations, we believe that postmenopausal corticosteroid users are now being reasonably well treated because bisphosphonate use was $61 \%$ and any prophylactic treatment was $72 \%$ in 2005 . The high use of prophylaxis in females probably reflects that doctors and pharmacists are finally recognizing this patient group. Although it is difficult to define an absolute standard of care, recognising contraindications as well as patient refusal or intolerance, we believe that in our population there is probably scope for further absolute improvement in the use of bisphosphonates of approximately $10-20 \%$ in females and $30-40 \%$ in males.

There are multiple reasons why patients are not receiving prophylaxis as recently highlighted [11]. Clearly, efforts are required to address the undertreatment and there have been interventions educating patients, pharmacists, GPs and specialists which demonstrated an improvement in CIOP management $[5,12]$. We are prospectively investigating the effect of a pharmacist recommendation to GPs whose patients require prophylaxis. Importantly, doctors are required to list specific reasons why their patients are not prescribed bisphosphonates, which we hope will allow a standard of care to be defined so future audits can assess if CIOP prevention is satisfactory.

\section{Conclusion}

We have found that in patients requiring prophylaxis with bisphosphonates according to Dutch guidelines, prevention was received in $54 \%$ of the cases in 2005 . It is clear that there has been a significant improvement in the treatment of women who now appear to be reasonably well treated. However, men are still not receiving prevention to the same degree as women, and it is now time to focus our attention and address this issue to identify if they are genuinely neglected.

Acknowledgment We would like to thank the pharmacists who provided data from their pharmacies.

\section{References}

1. Canalis E, Bilezikian JP, Angeli A, Giustina A (2004) Perspectives on glucocorticoid-induced osteoporosis. Bone 34:593-598

2. Lukert BP, Raisz LG (1990) Glucocorticoid-induced osteoporosis: pathogenesis and management. Ann Intern Med 112:352364

3. Curtis JR, Westfall AO, Allison JJ et al (2005) Longitudinal patterns in the prevention of osteoporosis in glucocorticoid-treated patients. Arthritis Rheum 52:2485-2494

4. Feldstein AC, Elmer PJ, Nichols GA, Herson M (2005) Practice patterns in patients at risk for glucocorticoid-induced osteoporosis. Osteoporos Int 16:2168-2174

5. Naunton M, Peterson GM, Jones G, Griffin GM, Bleasel MD (2004) Multifaceted educational program increases prescribing of preventive medication for corticosteroid induced osteoporosis. J Rheumatol 31:550-556

6. Osteoporose Tweede Herziene Richtlijn [Osteoporosis, Second Revised Guideline] (2002) ISBN 90-76906-23-8, 2nd ed. Utrecht: Van Zuiden Communications B.V.

7. World Health Organization. The WHO Collaborating Centre for Drug Statistics Methodology: http://www.whocc.no/atcddd/ (accessed February 2006)

8. Saag KG, Gehlbach SH, Curtis JR, Youket TE, Worley K, Lange JL (2006) Trends in prevention of glucocorticoid-induced osteoporosis. J Rheumatol 33:1651-1657

9. van Staa TP, Leufkens HG, Abenhaim L, Begaud B, Zhang B, Cooper C (2000) Use of oral corticosteroids in the United Kingdom. QJM 93:105-111

10. Walsh LJ, Wong CA, Pringle M, Tattersfield AE (1996) Use of oral corticosteroids in the community and the prevention of secondary osteoporosis: a cross sectional study. BMJ 313:344-346

11. Yood RA (2006) Prevention of glucocorticoid-induced osteoporosis: why are we doing so poorly? J Rheumatol 33:1461-1463

12. Newman ED, Matzko CK, Olenginski TP et al (2006) Glucocorticoid-Induced Osteoporosis Program (GIOP): a novel, comprehensive, and highly successful care program with improved outcomes at 1 year. Osteoporos Int 17:1428-1434 